\title{
Technology of MU Timekeeping Testing Based on Digital Transfer in Time Domain
}

\author{
Hu Juan ${ }^{1, \text { a }}$, LIU Kun ${ }^{2, b}$, Al Bing $^{3, c}$, SHI Qiang ${ }^{4, ~ d ~}$ \\ ${ }^{1}$ Sichuan Electric Power Corporation Maintenance Company Chengdu, China \\ ${ }^{2}$ Sichuan Electric Power Corporation Metering Center Chengdu, China \\ ${ }^{3}$ Sichuan Electric Power Corporation Metering Center Chengdu, China \\ ${ }^{4}$ Sichuan Electric Power Corporation Metering Center Chengdu, China \\ a21540772@qq.com, ${ }^{\text {b } 89195965 @ q q . c o m, ~}{ }^{\text {c5 } 56462972 @ q q . c o m, ~}{ }^{\text {dawaityou1986@gmail.com }}$
}

\begin{abstract}
Keywords:merging unit testing, digital transfer in time domain, timekeeping testing, phase measure Abstract. Merging unit timekeeping performance in the loss of synchronization signal, directly affect the synchronous acquisition of merging unit. At present, the test is only a test of pure time error. Further, the time error and the relationship between the synchronous sampling was not detected. This article proposed a merging unit timekeeping testing technology based on phase angle changes in time domain. Through a large number of test show that the proposed scheme, high accuracy, stable and feasible, and can be applied to the production.
\end{abstract}

\section{Introduction}

At present, the development of digital substation and smart substations have achieved a high level in the the project application. with the large-scale application of electronic transformers, transformer including the traditional merging unit (MU) mode, the merging unit has officially become intelligent electronic device of intelligent and digital substation. With the release of the $\mathrm{S}$ Q/GDW 441-2010 "intelligent substation protection technical specifications" and Q/GDW 396-2009 "IEC61850 protection engineering application model" ,SMV packet jitter, precision punctuality time characteristics have been made clearer specification [1] [2]. In the current situation, the need for accurate quantitative test merging unit for digital signal processing capabilities in case of loss of synchronization signals is urgent.

\section{Introduction to digital transfer in time domain}

Although, the State Grid Corporation requires that timekeeping ability after merging unit losing the sync signal must be no more than $4 \mathrm{uS}$ in 10 minutes, but at home and abroad we have no clear technical solutions to test the timekeeping ability of merge unit, which often characterized by some of the messages, or a pulse signal from the merging unit to test its timekeeping ability, but the way these uses can not reflect the timekeeping ability of merging unit essentially, merely reflect the handling pattern, deeper impact of the timekeeping module error on the synchronous sampling is not detectied, which leaving hidden dangers.

the essence of timekeeping ability of merging unit should be that delivery of the input signal for the transmission time acquisition and control in the case of losing synchronous signal should be a direct response to changes in the phase angle of the signal in the time domain, and timekeeping error on phase is often more concerned by the application of secondary equipment. Since the digital sampling value in accordance with the packet is transmitted as discrete points, so the ability to directly observe the reaction in synchronous digital phase angle variation in the time domain.

\section{Timekeeping testing of merging unit}

According to the input signal ,the merging unit is divided into different transformer interface with 
traditional analog input type which showed in figure 1 and digital interfaces with electronic transformer input type which showed in Figure 2.

Testing instrument receive GPS signals from the synchronization module and verify time, and generates its own sampling pulses used in the input signal as shown in Figure 3 (A), to power-frequency signal samples. In the GPS integer second time, samples sample R0 can be obtained guaranteed, including the sampling interval Ts1, continuous acquisition obtaining samples R1, R2 ....., shown in Fig 3 (B) for the entire time in seconds appears GPS, and Fig. 3 (C) sampling time for the tester, R0 sample corresponds exactly to the entire second moment in time. Fig. 3 (A) of the input signal to the starting time of R0, continuous acquisition of fixed time window (eg $80 \mathrm{~ms}$ ) of the sample, which undergoes hanning window and Fourier filtering phase of the signal can be obtained [10].

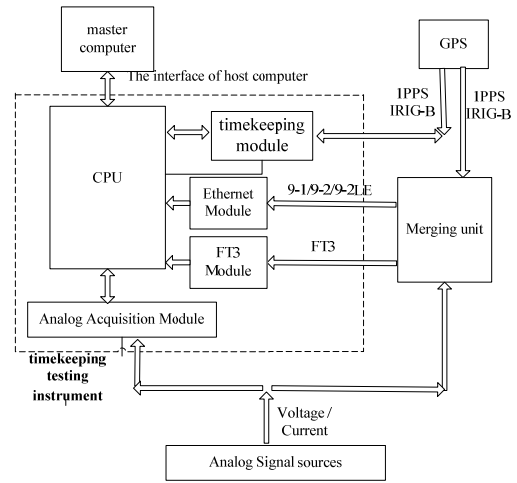

Fig 1 timekeeping test of MU of analog input

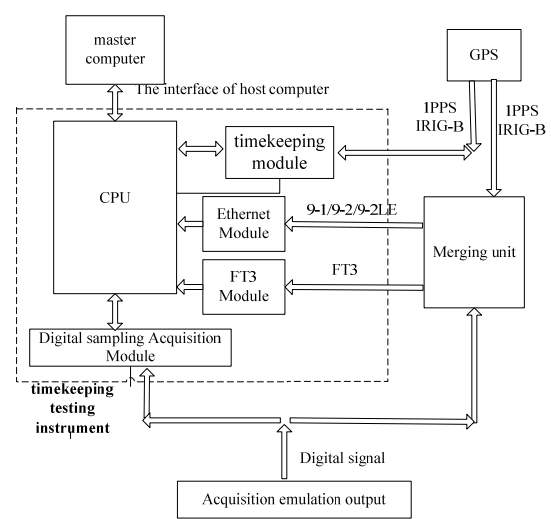

Fig 2 timekeeping test of MU of digital input

Hanning window (Hanning) can be seen as three rectangular time window spectrum sum, or a three sinc $(\mathrm{t})$ type and function of the main lobe widening and reduce side lobe is significantly reduced, decreasing from leakage standpoint Hanning than the rectangular window, you can eliminate high-frequency interference and leakage can be applied to non-periodic continuous signal.

When does not receive GPS, the merging unit is still in accordance with the time interval Ts2 during sampling, but this time the sampling pulse is generated based entirely on their own clock signal,it is not reliable. The sample counter is still a new sample for each transmitted data set, increased by one, in the entire second time inversion zero. As there is a certain error between the the merging unit time and the GPS time. as shown in Fig 3 (E), the counter for the sample T0 corresponding to the starting moment is not and GPS with full second time the emergence of the presence of a defensive error. Similarly with T0 as a starting time, and the reference amount to obtain successive samples of the same number of time windows, can obtain the phase of the signal. 3 (A), the combination of the frequency of the current signal, you can get defensive error $\neq 0$, as shown compensated electronic transformer rated phase offset remains unchanged, then $t_{d e l}=\frac{\varphi_{d e l}}{2 \pi f}$. 


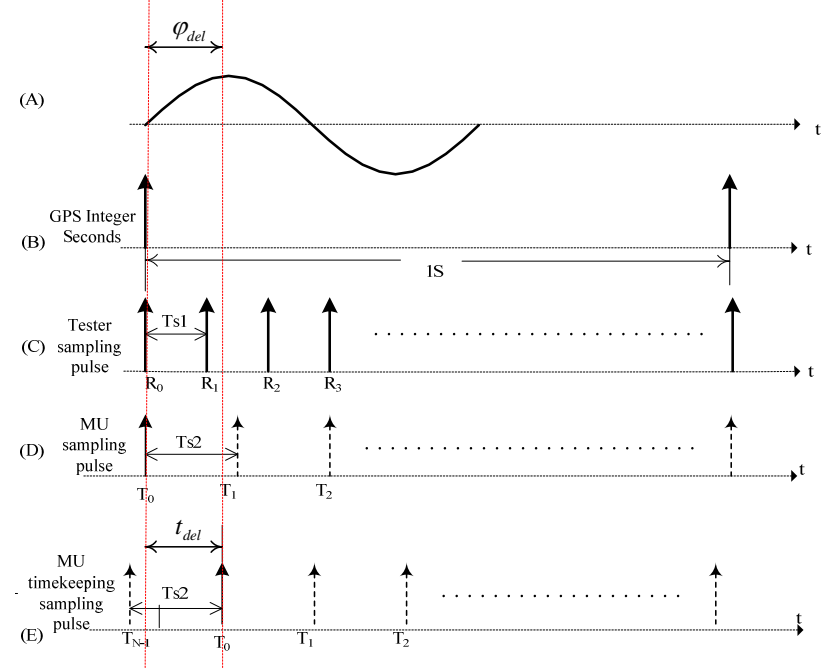

Fig 3 timekeeping accuracy of MU

\section{Time accuracy testing in the loss of synchronization signal}

Merging unit of analog input and digital input have been searched in this paper. analog input program merging unit 1 , the merging unit of rated phase offset is -3 degrees 17 minutes 24 seconds, the output protocol is IEC61850-9-1, the sampling rate is 200 points per cycle, when the GPS signal is switched off, the accuracy of the phase and time have been shown in Table 1.

Table 1 timekeeping testing of Merging unit of analog input in the loss of GPS signal

\begin{tabular}{|c|c|c|c|c|c|c|c|c|c|c|c|}
\hline & \multirow{2}{*}{$\begin{array}{c}\text { Synchronization } \\
\text { time }(\mathrm{m})\end{array}$} & \multicolumn{10}{|c|}{ Timekeeping period $(\mathrm{m})$} \\
\hline & & 1 & 2 & 3 & 4 & 5 & 6 & 7 & 8 & 9 & 10 \\
\hline $\begin{array}{l}\text { Phase error } \\
(\mathrm{m})\end{array}$ & 0 & -2.15 & -4.35 & -6.23 & -8.30 & -10.38 & -12.40 & -14.27 & -16.58 & -18.58 & -20.65 \\
\hline $\begin{array}{c}\text { Time } \\
\text { accuracy } \\
\text { (us) }\end{array}$ & 0 & 1.99 & 4.03 & 5.77 & 7.69 & 9.61 & 11.48 & 13.21 & 15.35 & 17.20 & 19.12 \\
\hline
\end{tabular}

when the 1PPS signal is switched off, the accuracy of the phase and time have been shown in Table 2.

Table 2 timekeeping testing of Merging unit of analog input in the loss of GPS signal

\begin{tabular}{c|c|c|c|c|c|c|c|c|c|c|c}
\hline & Synchronization & \multicolumn{9}{c|}{ Timekeeping period (m) } \\
\cline { 3 - 11 } & time(m) & 1 & 2 & 3 & 4 & 5 & 6 & 7 & 8 & 9 & 10 \\
\hline $\begin{array}{c}\text { Phase error } \\
(\mathrm{m})\end{array}$ & 0 & -2.11 & -4.40 & -6.18 & -8.05 & -10.03 & -12.14 & -13.97 & -16.01 & -17.86 & -19.77 \\
\hline $\begin{array}{c}\text { Time } \\
\text { accuracy } \\
\text { (us) }\end{array}$ & 0 & 1.95 & 4.07 & 5.72 & 7.45 & 9.29 & 11.24 & 12.94 & 14.82 & 16.54 & 18.31 \\
\hline
\end{tabular}

We found that the accuracy of the first MU's punctuality is worse than the second, in-depth understanding of discovery, the first MU uses ordinary precision SMD crystal, and adjust the synchronization signal tracking algorithm is not perfect. The TCXO second MU adopted, high accuracy and tracking adjustment algorithm is tested, and the accuracy of its defensive meet the requirements. Two MU for punctuality and precision GPS 1PPS signal, the difference 10 minutes, respectively, and in $0.81 \mathrm{uS} 0.54 \mathrm{uS}$, description of the type of the signal when the MU observe no effect on accuracy.

\section{Conclusion}

Based on the relationship between the phase and time in the digital time-domain transfer of merging unit, this paper proposed a merging unit synchronization keep testing technology,which test 
merging unit's timekeeping ability through the synchronous phase angle this technology, receiving clock from the GPS, can be compatible with merging unit synchronization keep merging unit of analog input and digital input .

This technology can test the characteristics of the merging unit effectively, and establish a theoretical basis for the large-scale promotion of intelligent substation.

\section{References}

[1] ZhouLi, Yinfeng.Analysis and Experiment of Transparent Clock in Time Synchronization System of Smart Substation[J]. Automation of Electric Power Systems,2012(06):106-111.

[2] ZHAO Hongda. Power time synchronization system and its accuracy monitoring[J]. Telecommunications for Electric Power System,2008(04):36-38.

[3] XU Zhiqiang,LEI Yutian,ZHANG Keren,LU Jun.An Approach of Time Measurement for Intelligent Components in Smart Substations[J]. Power System Technology,2011(12):8-13.

[4] IEC 60044-8:2002(E),Instrument Transformers-Part 8:Electronic Current Transformers[S].

[5] IEC61850 IEC. 9-1 Communication networks and systems in substations, part 9-1: Specific Communication Service Mapping (SCSM)-sampled values over multi-drop point link, 2003.

[6] IEC61850 IEC. 9-2 Communication networks and systems in substations, part 9-2: Specific Communication Service Mapping (SCSM)-sampled values over ISO[J]. IEC8802-3, 2004.

[7] LI Cheng,YUAN Yubo,LUO Qiang. Research on Interfacing Technology for Digital Protection Based on ECT/EVT[J]. Power System Technology,2007,31(9):84-87.

[8] Gong Jian,PENG Hui,QIAO Lufeng,ZHU Yong.FPGA Design and Implementation of Standard 802.3 Ethernet MAC Controller[J]. Journal of Military Communications Technology. 2005(04):21-24.

[9] XU Ke,DOU Xiaobo,HU Minqiang,ZHENG Jianyong. Microprocessor excitation regulator based on ARM and VxWorks[J]. Electric Power Automation Equipment, 2007(03):73-76.

[10]ZHOU Fuchao,WANG Zhiyong,JU Zipei.The Virtual Spectrum Analysis Instrument Based on LabVIEW[J]. Chinese Journal of Scientific Instrument. 2002(S2):741-743.

[11]JIANG Yuechun,TENG Zhaosheng,WANG Kenin,YANG Yuxiang. Electric Power Remote Monitoring and Management System Based on VB and LabView [J]. Instrument Technique and Sensor,2004(02):16-17. 